\title{
The DLR MiroSurge - A Robotic System for Surgery
}

\author{
Rainer Konietschke, Ulrich Hagn, Mathias Nickl, Stefan Jörg, Andreas Tobergte, Georg Passig, \\ Ulrich Seibold, Luc Le-Tien, Bernhard Kübler, Martin Gröger, Florian Fröhlich, Christian Rink, \\ Alin Albu-Schäffer, Markus Grebenstein, Tobias Ortmaier, and Gerd Hirzinger
}

\begin{abstract}
This video presents the in-house developed DLR MiroSurge robotic system for surgery. As shown, the system is suitable for both minimally invasive and open surgery. Essential part of the system is the MIRO robot: The soft robotics feature enables intuitive interaction with the robot.

In the presented minimally invasive robotic setup three MIROs guide an endoscopic stereo camera and two endoscopic instruments with force feedback sensors. The master console for teleoperation consists of an autostereoscopic monitor and force reflecting input devices for both hands.

Versatility is shown with two additional applications: For assistance in manual minimally invasive surgery a MIRO robot automatically guides the endoscope such that the surgical instrument is always in view. In a biopsy application the MIRO robot is positioning the needle with navigation system support.
\end{abstract}

\section{INTRODUCTION}

This video presents an in-house developed robotic system by the DLR (German Aerospace Center) designed for surgical applications named MiroSurge [1], see Fig. 2 and 3. The first part of the video shows the system in a minimally invasive surgery (MIS) setup, the second part presents the system's versatility in additional applications.

The paper is organized as follows: according to the sections in the video, firstly the DLR soft ("hands-on") robotics approach is presented (Sec. II). Subsequently, the preoperative planning and assisted setup procedure is briefly discussed (Sec. III), followed by a short description of the robotic MIS setup including the components of the force reflecting input console (Sec. IV). After the presentation of two additional applications to show MIRO's versatility (Sec. V), the paper concludes in Sec. VI with a short summary and outlook.

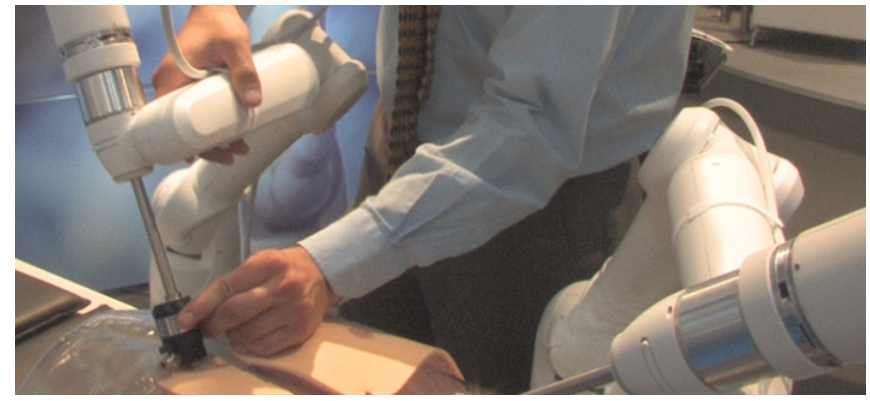

Fig. 1. Soft robotics enables hands-on the MIRO for insertion of the minimally invasive instrument into the patient.

The authors are affiliated with the Institute of Robotics and Mechatronics, German Aerospace Center (DLR), Wessling, Germany, rainer.konietschkeddlr.de

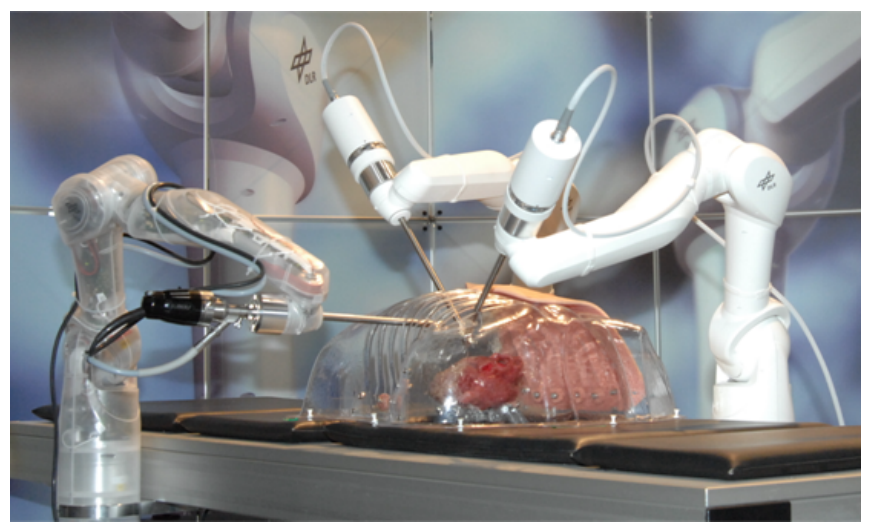

Fig. 2. Slave unit of the DLR MiroSurge telesurgery system. White MIROs carrying force/torque sensing MIS instruments (cp. Sec. IV) for bimanual manipulation; semi-transparent MIRO guiding a stereo endoscope.

\section{Soft ROBOtICS}

Aside from classic tele-robotics control, a soft ("handson") robotics feature is integrated into the workflow of the minimally invasive teleoperation: since all joint units of MIRO integrate both position and torque sensors, the robot can be used in impedance-controlled mode, allowing sensitive movements of the robot effected by the surgeon. Therewith, shared autonomy is possible: The orientation of the robot's end effector remains constant due to programmed high rotational stiffness, while its position can be adjusted by the surgeon simply by touching and moving the robot arm. Furthermore, the seven degrees of freedom (DoF) design of the MIRO robot arms allows for flexible configuration in the Nullspace. Due to the soft robotics capabilities, the MIS instrument can easily be inserted into the patient with constant tip rotation, see Fig. 1.

\section{OPTIMIZED SETUP}

The positions of the insertion points and robot bases are preoperatively planned and optimized, assisted by optimization algorithms that take into account the robot kinematics (avoidance of e.g. collisions, joint limits, singularities). A contact free surface-based registration of the patient is integrated into the workflow. The AutoPointer [2] then automatically projects the optimized data onto the patient. This way, the planned optimal setup can be conveniently transferred into the reality. 


\section{DeXTROUS TELESURGERY WITH FORCE FEEDBACK}

In the minimally invasive setup [4], three MIROs are involved: one is guiding an endoscopic stereo camera (Richard Wolf, Inc., Knittlingen, Germany), two more are guiding articulated and sensorized DLR MIS instruments for left and right hand manipulation. The master console is equipped with an autostereoscopic monitor (SeeFront, Inc., Hamburg, Germany) and force reflecting input devices (omega.7 by ForceDimension, Inc., Lausanne, Switzerland). See Tab. I for more details.

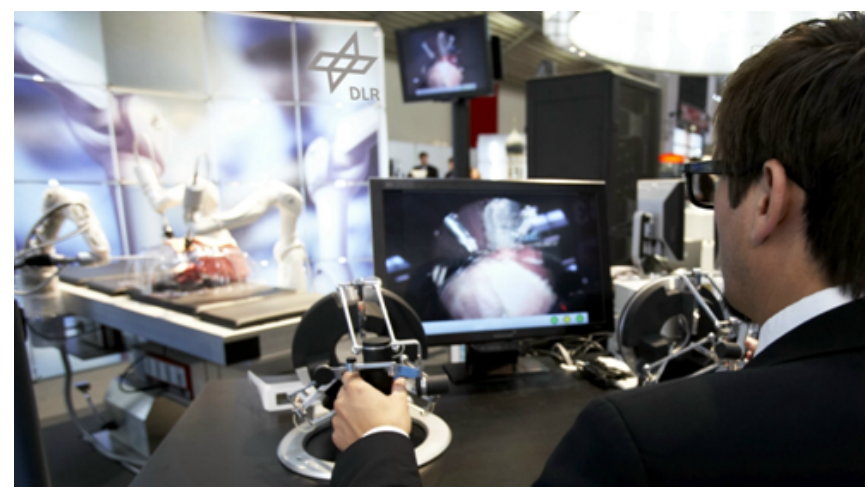

Fig. 3. The DLR MiroSurge robotic system for surgery. Depiction of the minimally invasive robotic surgery scenario. In the foreground: input console with force reflecting input devices (left and right hand, cp. Sec. IV) and $3 \mathrm{D}$ vision. In the background: slave unit with three MIRO robots (cp. Fig. 2).

The articulated MIS instruments are equipped with a six plus one DoF force/torque sensor measuring the contact situation as well as the gripping force. Two additional DoF inside the patient allow for dextrous telesurgery with full manipulability inside the patient and force feedback [6], [3].

During teleoperation mode, Nullspace motion maximizes the distance between the robots. At this point, the minimally invasive robotic surgery system with force feedback is functional and integrated.

\section{VERSATILITY}

To achieve versatility, the robotic system is optimized and designed for various applications in minimally invasive and open surgery [5]. For example, in a one-arm application for assisted minimally invasive manual surgery, the MIRO robot can be used to automatically guide the endoscope such that the instruments are always in view. A biopsy application was implemented with the KineMedic robot, a previous prototype. As shown in the video, a navigation system is used in the control loop to position the robot tip according to the preoperative biopsy planning.

\section{SUMMARY}

The referred video shows the DLR MiroSurge robotic surgery system. Soft robotics is highlighted with hands-on and shared autonomy. The application of minimally invasive surgery with bimanual force feedback is presented.

Furthermore, the system versatility is shown to allow additional applications also in open surgery. As future work, new
TABLE I

COMPONENTS OF THE DLR TELEROBOTIC SYSTEM FOR MIS

\begin{tabular}{|c|c|}
\hline Component & Parameters (per single component) \\
\hline \multicolumn{2}{|l|}{ Telemanipulators: } \\
\hline 3 DLR MIROs & $\begin{array}{ll}- & \text { } 7 \mathrm{DoF} \text { (redundant kinematics, com- } \\
\text { parable to the human arm) } \\
\text { - } \\
\text { weight }<10 \mathrm{~kg} \text { (capable for sin- } \\
\text { gle-person handling) } \\
\text { - } \\
\text { payload: } 30 \mathrm{~N} \text { (max. extended arm } \\
\text { position) } \\
\text { kinematic length: } 760 \mathrm{~mm} \text { (base } \\
\text { joint to flange) } \\
\text { position, torque, and impedance } \\
\text { control modes } \\
\text { control cycle: } 3 \cdot 10^{3} \mathrm{~Hz} \text { (sensor } \\
\text { monitoring, signal generation, and } \\
\text { transmissions) } \\
\text { max. velocity }>0.25 \mathrm{~m} / \mathrm{s}\end{array}$ \\
\hline \multicolumn{2}{|l|}{ Instruments: } \\
\hline 2 DLR MIS instruments & $\begin{array}{ll}- & 2 \text { articulated wrist joints } \\
- & 1 \text { articulated functional DoF } \\
- & 6 \text { DoF force/torque sensor (interac- } \\
& \text { tions) } \\
- & 1 \text { DoF gripping force sensor } \\
- & \text { endoscopic stereo camera }\end{array}$ \\
\hline \multicolumn{2}{|l|}{ Control interface: } \\
\hline 2 ForceDimension omega.7 & 6+1 DoF (3+1 DoF active) \\
\hline \multicolumn{2}{|l|}{ Display: } \\
\hline SeeFront 3D & autostereoscopic 3D display \\
\hline
\end{tabular}

applications are investigated, e.g. robotic laser osteotomy. Furthermore, motion compensation for operation on the virtually still standing heart in minimally invasive robotic surgery is envisioned.

\section{REFERENCES}

[1] Ulrich Hagn, Mathias Nickl, Stefan Jörg, Georg Passig, Thomas Bahls, Alexander Nothhelfer, Franz Hacker, Luc Le-Tien, Alin AlbuSchäffer, Rainer Konietschke, Markus Grebenstein, Rebecca Warpup, Robert Haslinger, Mirco Frommberger, and Gerd Hirzinger. The DLR MIRO: a versatile lightweight robot for surgical applications. Industrial Robot: An International Journal, 35(4):324-336, 2008. DOI: 10.1108/01439910810876427.

[2] Rainer Konietschke, A. Knöferle, and Gerd Hirzinger. The AutoPointer: A new augmented-reality device for transfer of planning data into the operating room. In Proceedings of the 21st International Congress and Exhibition of Computer Assisted Radiology and Surgery (CARS), Berlin, Germany, June 2007.

[3] Bernhard Kübler, Ulrich Seibold, and Gerd Hirzinger. Development of actuated and sensor integrated forceps for minimally invasive robotic surgery. International Journal of Medical Robotics and Computer Assisted Surgery, 1(3):96-107, 2005. DOI: 10.1002/rcs.33.

[4] Tobias Ortmaier, Barbara Deml, Bernhard Kübler, Georg Passig, Detlef Reintsema, and Ulrich Seibold. Advances in Telerobotics, volume 31 of Springer Tracts in Advanced Robotics (STAR), chapter Robot Assisted Force Feedback Surgery, pages 361-379. Springer, Berlin/Heidelberg, 2007. DOI: 10.1007/978-3-540-71364-7_22.

[5] Tobias Ortmaier, Holger Weiss, Ulrich Hagn, Markus Grebenstein, Mathias Nickl, Alin Albu-Schäffer, Christian Ott, Stefan Jörg, Rainer Konietschke, Luc Le-Tien, and Gerd Hirzinger. A hands-on-robot for accurate placement of pedicle screws. In Proceedings of the IEEE International Conference on Robotics and Automation (ICRA), pages 4179-4186, Orlando, Florida, USA, May 2006.

[6] Ulrich Seibold, Bernhard Kübler, and Gerd Hirzinger. Medical Robotics, chapter Chapter 28 - Prototypic force feedback instrument for minimally invasive robotic surgery, pages 377-400. International Journal of Advanced Robotic Systems, Vienna, Austria, 2008. ISBN13: $978-3-902613-18-9$. 